\title{
On unimodality problems in Pascal's triangle *
}

\author{
Xun-Tuan Su and Yi Wang ${ }^{\dagger}$ \\ Department of Applied Mathematics \\ Dalian University of Technology \\ Dalian 116024, P. R. China \\ suxuntuan@yahoo.com.cn \\ wangyi@dlut.edu.cn
}

Submitted: Jan 23, 2008; Accepted: Aug 28, 2008; Published: Sep 8, 2008

Mathematics Subject Classification: 05A10, 05A20

\begin{abstract}
Many sequences of binomial coefficients share various unimodality properties. In this paper we consider the unimodality problem of a sequence of binomial coefficients located in a ray or a transversal of the Pascal triangle. Our results give in particular an affirmative answer to a conjecture of Belbachir et al which asserts that such a sequence of binomial coefficients must be unimodal. We also propose two more general conjectures.
\end{abstract}

\section{Introduction}

Let $a_{0}, a_{1}, a_{2}, \ldots$ be a sequence of nonnegative numbers. It is called unimodal if $a_{0} \leq$ $a_{1} \leq \cdots \leq a_{m-1} \leq a_{m} \geq a_{m+1} \geq \cdots$ for some $m$ (such an integer $m$ is called a mode of the sequence). In particular, a monotone (increasing or decreasing) sequence is known as unimodal. The sequence is called concave (resp. convex) if for $i \geq 1, a_{i-1}+a_{i+1} \leq 2 a_{i}$ (resp. $a_{i-1}+a_{i+1} \geq 2 a_{i}$ ). The sequence is called log-concave (resp. log-convex) if for all $i \geq 1, a_{i-1} a_{i+1} \leq a_{i}^{2}$ (resp. $a_{i-1} a_{i+1} \geq a_{i}^{2}$ ). By the arithmetic-geometric mean inequality, the concavity implies the log-concavity (the log-convexity implies the convexity). For a sequence $\left\{a_{i}\right\}$ of positive numbers, it is log-concave (resp. log-convex) if and only if the sequence $\left\{a_{i+1} / a_{i}\right\}$ is decreasing (resp. increasing), and so the log-concavity implies the unimodality. The unimodality problems, including concavity (convexity) and logconcavity (log-convexity), arise naturally in many branches of mathematics. For details, see $[3,4,13,17,18,19,21,22]$ about the unimodality and log-concavity and $[7,10]$ about the log-convexity.

${ }^{*}$ Partially supported by the National Science Foundation of China under Grant No.10771027.

${ }^{\dagger}$ Corresponding author. 
Many sequences of binomial coefficients share various unimodality properties. For example, the sequence $\left\{\left(\begin{array}{l}n \\ k\end{array}\right)\right\}_{k=0}^{n}$ is unimodal and log-concave in $k$. On the other hand, the sequence $\left\{\left(\begin{array}{l}n \\ k\end{array}\right)\right\}_{n=k}^{+\infty}$ is increasing, log-concave and convex in $n$ (see Comtet [5] for example). As usual, let $\left(\begin{array}{l}n \\ k\end{array}\right)=0$ unless $0 \leq k \leq n$. Tanny and Zuker $[14,15]$ showed the unimodality and log-concavity of the binomial sequences $\left\{\left(\begin{array}{c}n_{0}-i \\ i\end{array}\right)\right\}_{i}$ and $\left\{\left(\begin{array}{c}n_{0}-i d \\ i\end{array}\right)\right\}_{i}$. Very recently, Belbachir et al [1] showed the unimodality and log-concavity of the binomial sequence $\left\{\left(\begin{array}{c}n_{0}+i \\ i d\end{array}\right)\right\}_{i}$. They further proposed the following.

Conjecture 1 ([1, Conjecture 1$])$. Let $\left(\begin{array}{l}n \\ k\end{array}\right)$ be a fixed element of the Pascal triangle crossed by a ray. The sequence of binomial coefficients located along this ray is unimodal.

$\left(\begin{array}{l}0 \\ 0\end{array}\right)$

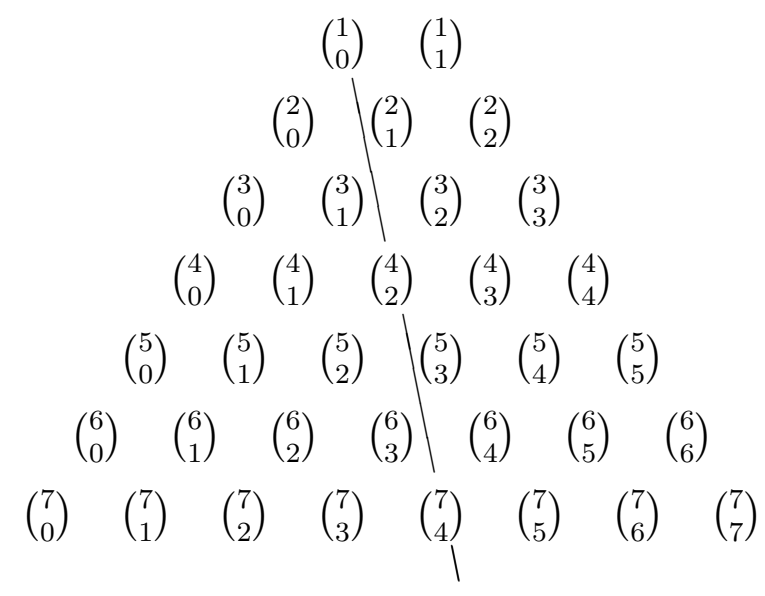

Figure 1: a ray with $d=3$ and $\delta=2$.

The object of this paper is to study the unimodality problem of a sequence of binomial coefficients located in a ray or a transversal of the Pascal triangle. Let $\left\{\left(\begin{array}{l}n_{i} \\ k_{i}\end{array}\right)\right\}_{i \geq 0}$ be such a sequence. Then $\left\{n_{i}\right\}_{i \geq 0}$ and $\left\{k_{i}\right\}_{i \geq 0}$ form two arithmetic sequences (see Figure 1). Clearly, we may assume that the common difference of $\left\{n_{i}\right\}_{i \geq 0}$ is nonnegative (by changing the order of the sequence). For example, the sequence $\left\{\left(\begin{array}{c}n_{0}-i \\ i\end{array}\right)\right\}_{i=0}^{\left\lfloor\frac{n_{0}}{2}\right\rfloor}$ coincides with the sequence $\left\{\left(\begin{array}{c}n_{0}-\left\lfloor\frac{n_{0}}{2}\right\rfloor+i \\ \left\lfloor\frac{n_{0}}{2}\right\rfloor-i\end{array}\right)\right\}_{i=0}^{\left\lfloor\frac{n_{0}}{2}\right\rfloor}$ except for the order. On the other hand, the sequence $\left\{\left(\begin{array}{c}n_{i} \\ k_{i}\end{array}\right)\right\}_{i \geq 0}$ is the same as the sequence $\left\{\left(\begin{array}{c}n_{i} \\ n_{i}-k_{i}\end{array}\right)\right\}_{i \geq 0}$ by the symmetry of the binomial coefficients. So we may assume, without loss of generality, that the common difference of $\left\{k_{i}\right\}_{i \geq 0}$ is nonnegative. Thus it suffices to consider the unimodality of the sequence $\left\{\left(\begin{array}{c}n_{0}+i d \\ k_{0}+i \delta\end{array}\right)\right\}_{i \geq 0}$ for nonnegative integers $d$ and $\delta$. The following is the main result of this paper, which in particular, gives an affirmative answer to Conjecture 1.

Theorem 1. Let $n_{0}, k_{0}, d, \delta$ be four nonnegative integers and $n_{0} \geq k_{0}$. Define the sequence

$$
C_{i}=\left(\begin{array}{l}
n_{0}+i d \\
k_{0}+i \delta
\end{array}\right), \quad i=0,1,2, \ldots
$$


Then

(i) if $d=\delta>0$ or $\delta=0$, the sequence is increasing, convex and log-concave;

(ii) if $d<\delta$, the sequence is log-concave and therefore unimodal;

(iii) if $d>\delta>0$, the sequence is increasing, convex, and asymptotically log-convex (i.e., there exists a nonnegative integer $m$ such that $C_{m}, C_{m+1}, C_{m+2}, \ldots$ is log-convex).

This paper is organized as follows. In the next section, we prove Theorem 1. In Section 3, we present a combinatorial proof of the log-concavity in Theorem 1 (ii). In Section 4, we show more precise results about the asymptotically log-convexity for certain particular sequences of binomial coefficients in Theorem 1 (iii). Finally in Section 5, we propose some open problems and conjectures.

Throughout this paper we will denote by $\lfloor x\rfloor$ and $\lceil x\rceil$ the largest integer $\leq x$ and the smallest integer $\geq x$ respectively.

\section{The proof of Theorem 1}

The following result is folklore and we include a proof of it for completeness.

Lemma 1. If a sequence $\left\{a_{i}\right\}_{i \geq 0}$ of positive numbers is unimodal (resp. increasing, decreasing, concave, convex, log-concave, log-convex), then so is its subsequence $\left\{a_{n_{0}+i d}\right\}_{i \geq 0}$ for arbitrary fixed nonnegative integers $n_{0}$ and $d$.

Proof. We only consider the log-concavity case since the others are similar. Let $\left\{a_{i}\right\}_{i \geq 0}$ be a log-concave sequence of positive numbers. Then the sequence $\left\{a_{i-1} / a_{i}\right\}_{i \geq 0}$ is increasing. Hence $a_{j-1} / a_{j} \leq a_{k} / a_{k+1}$ for $1 \leq j \leq k$, i.e., $a_{j-1} a_{k+1} \leq a_{j} a_{k}$. Thus

$$
a_{n-d} a_{n+d} \leq a_{n-d+1} a_{n+d-1} \leq a_{n-d+2} a_{n+d-2} \leq \cdots \leq a_{n-1} a_{n+1} \leq a_{n}^{2},
$$

which implies that the sequence $\left\{a_{n_{0}+i d}\right\}_{i \geq 0}$ is log-concave.

The proof of Theorem 1. (i) If $\delta=0$, then $C_{i}=\left(\begin{array}{c}n_{0}+i d \\ k_{0}\end{array}\right)$. The sequence $\left(\begin{array}{c}i \\ k_{0}\end{array}\right)$ is increasing, convex and $\log$-concave in $i$, so is the sequence $C_{i}$ by Lemma 1 . The case $d=\delta$ is similar since $C_{i}=\left(\begin{array}{c}n_{0}+i d \\ n_{0}-k_{0}\end{array}\right)$.

(ii) To show the log-concavity of $\left\{C_{i}\right\}$ when $d<\delta$, it suffices to show that

$$
\left(\begin{array}{l}
n+d \\
k+\delta
\end{array}\right)\left(\begin{array}{l}
n-d \\
k-\delta
\end{array}\right) \leq\left(\begin{array}{l}
n \\
k
\end{array}\right)^{2}
$$

for $n \geq k$. Write

$$
\begin{aligned}
\left(\begin{array}{l}
n+d \\
k+\delta
\end{array}\right)\left(\begin{array}{l}
n-d \\
k-\delta
\end{array}\right) & =\frac{(n+d) !(n-d) !}{(n-k+d-\delta) !(k+\delta) !(n-k+\delta-d) !(k-\delta) !} \\
& =\left(\begin{array}{l}
n+d \\
n-k
\end{array}\right)\left(\begin{array}{l}
n-d \\
n-k
\end{array}\right) \frac{\left(\begin{array}{c}
n-k \\
\delta-d
\end{array}\right)}{\left(\begin{array}{c}
n-k+\delta-d \\
\delta-d
\end{array}\right)} \frac{\left(\begin{array}{l}
k-d \\
\delta-d
\end{array}\right)}{\left(\begin{array}{l}
k+\delta \\
\delta-d
\end{array}\right)} .
\end{aligned}
$$


Now $\left(\begin{array}{c}n-k \\ \delta-d\end{array}\right) \leq\left(\begin{array}{c}n-k+\delta-d \\ \delta-d\end{array}\right),\left(\begin{array}{c}k-d \\ \delta-d\end{array}\right) \leq\left(\begin{array}{c}k+\delta \\ \delta-d\end{array}\right)$ and $\left(\begin{array}{l}n+d \\ n-k\end{array}\right)\left(\begin{array}{l}n-d \\ n-k\end{array}\right) \leq\left(\begin{array}{c}n \\ n-k\end{array}\right)^{2}$ by (i). Hence

$$
\left(\begin{array}{l}
n+d \\
k+\delta
\end{array}\right)\left(\begin{array}{l}
n-d \\
k-\delta
\end{array}\right) \leq\left(\begin{array}{c}
n \\
n-k
\end{array}\right)^{2}=\left(\begin{array}{l}
n \\
k
\end{array}\right)^{2}
$$

as required.

(iii) Assume that $d>\delta>0$. By Vandermonde's convolution formula, we have

$$
\left(\begin{array}{l}
n+d \\
k+\delta
\end{array}\right)=\sum_{r+s=k+\delta}\left(\begin{array}{l}
n \\
r
\end{array}\right)\left(\begin{array}{l}
d \\
s
\end{array}\right) \geq\left(\begin{array}{l}
n \\
k
\end{array}\right)\left(\begin{array}{l}
d \\
\delta
\end{array}\right) \geq 2\left(\begin{array}{l}
n \\
k
\end{array}\right),
$$

which implies that $\left(\begin{array}{l}n+d \\ k+\delta\end{array}\right)>\left(\begin{array}{l}n \\ k\end{array}\right)$ and $\left(\begin{array}{l}n+d \\ k+\delta\end{array}\right)+\left(\begin{array}{l}n-d \\ k-\delta\end{array}\right) \geq 2\left(\begin{array}{l}n \\ k\end{array}\right)$. Hence the sequence $\left\{C_{i}\right\}$ is increasing and convex.

It remains to show that the sequence $\left\{C_{i}\right\}$ is asymptotically log-convex. Denote

$$
\Delta(i):=\left(\begin{array}{l}
n_{0}+(i+1) d \\
k_{0}+(i+1) \delta
\end{array}\right)\left(\begin{array}{l}
n_{0}+(i-1) d \\
k_{0}+(i-1) \delta
\end{array}\right)-\left(\begin{array}{c}
n_{0}+i d \\
k_{0}+i \delta
\end{array}\right)^{2} .
$$

Then we need to show that $\Delta(i)$ is positive for all sufficiently large $i$. Write

$$
\begin{aligned}
\Delta(i)= & \frac{\left(n_{0}+i d\right) !\left[n_{0}+(i-1) d\right] !}{\left(k_{0}+i \delta\right) !\left[k_{0}+(i+1) \delta\right] !\left[n_{0}-k_{0}+i(d-\delta)\right] !\left[n_{0}-k_{0}+(i+1)(d-\delta)\right] !} \\
& \times\left\{\prod_{j=1}^{d}\left(n_{0}+i d+j\right) \prod_{j=1}^{d-\delta}\left[n_{0}-k_{0}+(i-1)(d-\delta)+j\right] \prod_{j=1}^{\delta}\left[k_{0}+(i-1) \delta+j\right]\right. \\
& \left.-\prod_{j=1}^{d}\left[n_{0}+(i-1) d+j\right] \prod_{j=1}^{d-\delta}\left[n_{0}-k_{0}+i(d-\delta)+j\right] \prod_{j=1}^{\delta}\left(k_{0}+i \delta+j\right)\right\} \\
= & \frac{\left(n_{0}+i d\right) !\left[n_{0}+(i-1) d\right] ! d^{d} \delta^{\delta}(d-\delta)^{(d-\delta)}}{\left(k_{0}+i \delta\right) !\left[k_{0}+(i+1) \delta\right] !\left[n_{0}-k_{0}+i(d-\delta)\right] !\left[n_{0}-k_{0}+(i+1)(d-\delta)\right] !} P(i),
\end{aligned}
$$

where

$$
\begin{aligned}
P(i)= & \prod_{j=1}^{d}\left(i+\frac{n_{0}+j}{d}\right) \prod_{j=1}^{d-\delta}\left(i+\frac{n_{0}-k_{0}-d+\delta+j}{d-\delta}\right) \prod_{j=1}^{\delta}\left(i+\frac{k_{0}-\delta+j}{\delta}\right) \\
& -\prod_{j=1}^{d}\left(i+\frac{n_{0}-d+j}{d}\right) \prod_{j=1}^{d-\delta}\left(i+\frac{n_{0}-k_{0}+j}{d-\delta}\right) \prod_{j=1}^{\delta}\left(i+\frac{k_{0}+j}{\delta}\right) .
\end{aligned}
$$

Then it suffices to show that $P(i)$ is positive for sufficiently large $i$. Clearly, $P(i)$ can be viewed as a polynomial in $i$. So it suffices to show that the leading coefficient of $P(i)$ is positive.

Note that $P(i)$ is the difference of two monic polynomials of degree $2 d$. Hence its degree is less than $2 d$. Denote

$$
P(i)=a_{2 d-1} i^{2 d-1}+a_{2 d-2} i^{2 d-2}+\cdots .
$$


By Vieta's formula, we have

$$
\begin{aligned}
a_{2 d-1}= & -\left(\sum_{j=1}^{d} \frac{n_{0}+j}{d}+\sum_{j=1}^{d-\delta} \frac{n_{0}-k_{0}-d+\delta+j}{d-\delta}+\sum_{j=1}^{\delta} \frac{k_{0}-\delta+j}{\delta}\right) \\
& +\left(\sum_{j=1}^{d} \frac{n_{0}-d+j}{d}+\sum_{j=1}^{d-\delta} \frac{n_{0}-k_{0}+j}{d-\delta}+\sum_{j=1}^{\delta} \frac{k_{0}+j}{\delta}\right) \\
= & \sum_{j=1}^{d}\left(\frac{n_{0}-d+j}{d}-\frac{n_{0}+j}{d}\right) \\
& +\sum_{j=1}^{d-\delta}\left(\frac{n_{0}-k_{0}+j}{d-\delta}-\frac{n_{0}-k_{0}-d+\delta+j}{d-\delta}\right) \\
& +\sum_{j=1}^{\delta}\left(\frac{k_{0}+j}{\delta}-\frac{k_{0}-\delta+j}{\delta}\right) \\
= & \sum_{j=1}^{d}(-1)+\sum_{j=1}^{d-\delta} 1+\sum_{j=1}^{\delta} 1 \\
= & -d+(d-\delta)+\delta \\
= & 0 .
\end{aligned}
$$

Using the identity

$$
\sum_{1 \leq i<j \leq n} x_{i} x_{j}=\frac{1}{2}\left[\left(\sum_{i=1}^{n} x_{i}\right)^{2}-\sum_{i=1}^{n} x_{i}^{2}\right],
$$

we obtain again by Vieta's formula

$$
\begin{aligned}
a_{2 d-2}= & \frac{1}{2}\left[\left(\sum_{j=1}^{d} \frac{n_{0}+j}{d}+\sum_{j=1}^{d-\delta} \frac{n_{0}-k_{0}-d+\delta+j}{d-\delta}+\sum_{j=1}^{\delta} \frac{k_{0}-\delta+j}{\delta}\right)^{2}\right. \\
& \left.-\left(\sum_{j=1}^{d} \frac{n_{0}+j}{d}\right)^{2}-\left(\sum_{j=1}^{d-\delta} \frac{n_{0}-k_{0}-d+\delta+j}{d-\delta}\right)^{2}-\left(\sum_{j=1}^{\delta} \frac{k_{0}-\delta+j}{\delta}\right)^{2}\right] \\
& -\frac{1}{2}\left[\left(\sum_{j=1}^{d} \frac{n_{0}-d+j}{d}+\sum_{j=1}^{d-\delta} \frac{n_{0}-k_{0}+j}{d-\delta}+\sum_{j=1}^{\delta} \frac{k_{0}+j}{\delta}\right)^{2}\right. \\
& \left.-\left(\sum_{j=1}^{d} \frac{n_{0}-d+j}{d}\right)^{2}-\left(\sum_{j=1}^{d-\delta} \frac{n_{0}-k_{0}+j}{d-\delta}\right)^{2}-\left(\sum_{j=1}^{\delta} \frac{k_{0}+j}{d-\delta}\right)^{2}\right] .
\end{aligned}
$$


But $a_{2 d-1}=0$ implies

$$
\begin{aligned}
& \left(\sum_{j=1}^{d} \frac{n_{0}+j}{d}+\sum_{j=1}^{d-\delta} \frac{n_{0}-k_{0}-d+\delta+j}{d-\delta}+\sum_{j=1}^{\delta} \frac{k_{0}-\delta+j}{\delta}\right)^{2} \\
= & \left(\sum_{j=1}^{d} \frac{n_{0}-d+j}{d}+\sum_{j=1}^{d-\delta} \frac{n_{0}-k_{0}+j}{d-\delta}+\sum_{j=1}^{\delta} \frac{k_{0}+j}{\delta}\right)^{2},
\end{aligned}
$$

so we have

$$
\begin{aligned}
a_{2 d-2}= & \frac{1}{2} \sum_{j=1}^{d}\left[\left(\frac{n_{0}-d+j}{d}\right)^{2}-\left(\frac{n_{0}+j}{d}\right)^{2}\right] \\
& +\frac{1}{2} \sum_{j=1}^{d-\delta}\left[\left(\frac{n_{0}-k_{0}+j}{d-\delta}\right)^{2}-\left(\frac{n_{0}-k_{0}-d+\delta+j}{d-\delta}\right)^{2}\right] \\
& +\frac{1}{2} \sum_{j=1}^{\delta}\left[\left(\frac{k_{0}+j}{\delta}\right)^{2}-\left(\frac{k_{0}-\delta+j}{\delta}\right)^{2}\right] \\
= & -\frac{1}{2} \sum_{j=1}^{d} \frac{2 n_{0}-d+2 j}{d}+\frac{1}{2} \sum_{j=1}^{d-\delta} \frac{2\left(n_{0}-k_{0}\right)-(d-\delta)+2 j}{d-\delta}+\frac{1}{2} \sum_{j=1}^{\delta} \frac{2 k_{0}-\delta+2 j}{\delta} \\
= & -\frac{1}{2}\left(2 n_{0}+1\right)+\frac{1}{2}\left(2 n_{0}-2 k_{0}+1\right)+\frac{1}{2}\left(2 k_{0}+1\right) \\
= & \frac{1}{2} .
\end{aligned}
$$

Thus $P(i)$ is a polynomial of degree $2 d-2$ with positive leading coefficient, as desired. This completes the proof of the theorem.

\section{Combinatorial proof of the log-concavity}

In Section 2 we have investigated the unimodality of sequences of binomial coefficients by an algebraic approach. It is natural to ask for a combinatorial interpretation. Lattice path techniques have been shown to be useful in solving the unimodality problem. As an example, we present a combinatorial proof of Theorem 1 (ii) following Bóna and Sagan's technique in [2].

Let $\mathbb{Z}^{2}=\{(x, y): x, y \in \mathbb{Z}\}$ denote the two-dimensional integer lattice. A lattice path is a sequence $P_{1}, P_{2}, \ldots, P_{\ell}$ of lattice points on $\mathbb{Z}^{2}$. A southeastern lattice path is a lattice path in which each step goes one unit to the south or to the east. Denote by $P(n, k)$ the set of southeastern lattice paths from the point $(0, n-k)$ to the point $(k, 0)$. Clearly, the number of such paths is the binomial coefficient $\left(\begin{array}{l}n \\ k\end{array}\right)$.

Recall that, to show the log-concavity of $C_{i}=\left(\begin{array}{l}n_{0}+i d \\ k_{0}+i \delta\end{array}\right)$ where $n_{0} \geq k_{0}$ and $d<\delta$, it suffices to show $\left(\begin{array}{c}n+d \\ k+\delta\end{array}\right)\left(\begin{array}{l}n-d \\ k-\delta\end{array}\right) \leq\left(\begin{array}{l}n \\ k\end{array}\right)^{2}$ for $n \geq k$. Here we do this by constructing an injection

$$
\phi: P(n+d, k+\delta) \times P(n-d, k-\delta) \longrightarrow P(n, k) \times P(n, k) .
$$


Consider a path pair $(p, q) \in P(n+d, k+\delta) \times P(n-d, k-\delta)$. Then $p$ and $q$ must intersect. Let $I_{1}$ be the first intersection. For two points $P(a, b)$ and $Q(a, c)$ with the same $x$-coordinate, define their vertical distance to be $d_{v}(P, Q)=b-c$. Then the vertical distance from a point of $p$ to a point of $q$ starts at $2(\delta-d)$ for their initial points and ends at 0 for their intersection $I_{1}$. Thus there must be a pair of points $P \in p$ and $Q \in q$ before $I_{1}$ with $d_{v}(P, Q)=\delta-d$. Let $\left(P_{1}, Q_{1}\right)$ be the first such pair of points. Similarly, after the last intersection $I_{2}$ there must be a last pair of points $P_{2} \in p$ and $Q_{2} \in q$ with the horizontal distance $d_{h}\left(P_{2}, Q_{2}\right)=-\delta$ (the definition of $d_{h}$ is analogous to that of $\left.d_{v}\right)$. Now $p$ is divided by two points $P_{1}, P_{2}$ into three subpaths $p_{1}, p_{2}, p_{3}$ and $q$ is divided by $Q_{1}, Q_{2}$ into three subpaths $q_{1}, q_{2}, q_{3}$. Let $p_{1}^{\prime}$ be obtained by moving $p_{1}$ down to $Q_{1}$ south $\delta-d$ units and $p_{3}^{\prime}$ be obtained by moving $p_{3}$ right to $Q_{2}$ east $\delta$ units. Then we obtain a southeastern lattice path $p_{1}^{\prime} q_{2} p_{3}^{\prime}$ in $P(n, k)$. We can similarly obtain the second southeastern lattice path $q_{1}^{\prime} p_{2} q_{3}^{\prime}$ in $P(n, k)$, where $q_{1}^{\prime}$ is $q_{1}$ moved north $\delta-d$ units and $q_{3}^{\prime}$ is $q_{3}$ moved west $\delta$ units. Define $\phi(p, q)=\left(p_{1}^{\prime} q_{2} p_{3}^{\prime}, q_{1}^{\prime} p_{2} q_{3}^{\prime}\right)$. It is not difficult to verify that $\phi$ is the required injective. We omit the proof for brevity.
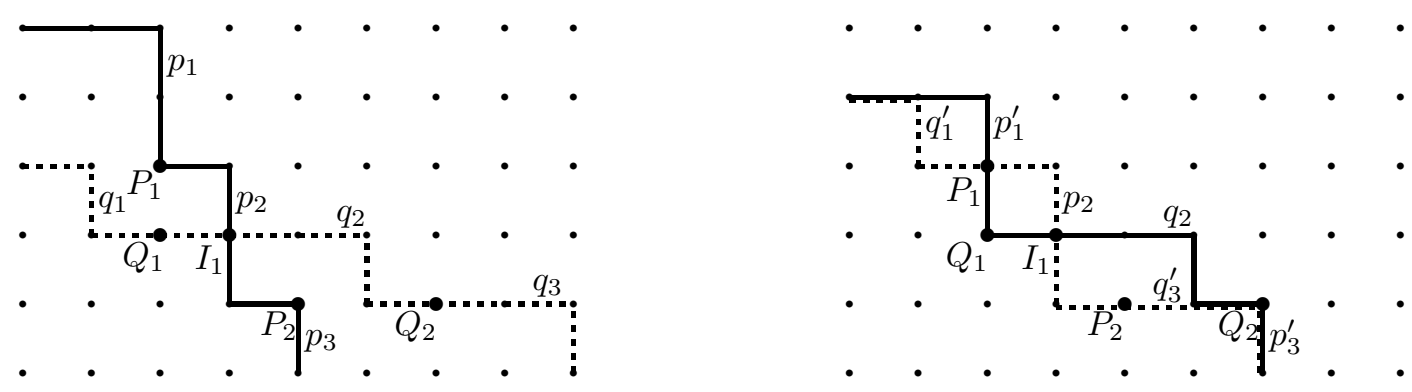

Figure 2: the constructing of $\phi$.

\section{Asymptotic behavior of the log-convexity}

Theorem 1 (iii) tells us that the sequence $C_{i}=\left(\begin{array}{l}n_{0}+i d \\ k_{0}+i \delta\end{array}\right)$ is asymptotically log-convex when $d>\delta>0$. We can say more for a certain particular sequence of binomial coefficients. For example, it is easy to verify that the central binomial coefficients $\left(\begin{array}{c}2 i \\ i\end{array}\right)$ is log-convex for $i \geq 0$ (see Liu and Wang [10] for a proof). In this section we give two generalizations of this result. The first one is that every sequence of binomial coefficients located along a ray with origin $\left(\begin{array}{l}0 \\ 0\end{array}\right)$ is log-convex.

Proposition 1. Let $d$ and $\delta$ be two positive integers and $d>\delta>0$. Then the sequence $\left\{\left(\begin{array}{l}\text { id } \\ i \delta\end{array}\right)\right\}_{i \geq 0}$ is log-convex.

Before showing Proposition 1, we first demonstrate two simple but useful facts.

Let $\alpha=\left(a_{1}, a_{2}, \ldots, a_{n}\right)$ and $\beta=\left(b_{1}, b_{2}, \ldots, b_{n}\right)$ be two $n$-tuples of real numbers. We say that $\alpha$ alternates left of $\beta$, denoted by $\alpha \preceq \beta$, if

$$
a_{1}^{*} \leq b_{1}^{*} \leq a_{2}^{*} \leq b_{2}^{*} \cdots \leq a_{n}^{*} \leq b_{n}^{*},
$$

where $a_{j}^{*}$ and $b_{j}^{*}$ are the $j$ th smallest elements of $\alpha$ and $\beta$, respectively. 
Fact 1 Let $f(x)$ be a nondecreasing function. If $\left(a_{1}, a_{2}, \ldots, a_{n}\right) \preceq\left(b_{1}, b_{2}, \ldots, b_{n}\right)$, then $\prod_{i=1}^{n} f\left(a_{i}\right) \leq \prod_{i=1}^{n} f\left(b_{i}\right)$.

Fact 2 Let $x_{1}, x_{2}, y_{1}, y_{2}$ be four positive numbers and $\frac{x_{1}}{y_{1}} \leq \frac{x_{2}}{y_{2}}$. Then $\frac{x_{1}}{y_{1}} \leq \frac{x_{1}+x_{2}}{y_{1}+y_{2}} \leq \frac{x_{2}}{y_{2}}$.

Proof of Proposition 1. By Lemma 1, we may assume, without loss of generality, that $d$ and $\delta$ are coprime. We need to show that

$$
\Delta(i):=\left(\begin{array}{c}
(i+1) d \\
(i+1) \delta
\end{array}\right)\left(\begin{array}{c}
(i-1) d \\
(i-1) \delta
\end{array}\right)-\left(\begin{array}{c}
i d \\
i \delta
\end{array}\right)^{2} \geq 0
$$

for all $i \geq 1$. Write

$$
\Delta(i)=\frac{(i d) ![(i-1) d] ! d^{d} \delta^{\delta}(d-\delta)^{(d-\delta)} \prod_{j=1}^{d}\left(i+\frac{j}{d}\right) \prod_{j=1}^{\delta}\left(i+\frac{j}{\delta}\right) \prod_{j=1}^{d-\delta}\left(i+\frac{j}{d-\delta}\right)}{(i \delta) ![(i+1) \delta] ![i(d-\delta)] ![(i+1)(d-\delta)] !} Q(i),
$$

where

$$
Q(i)=\prod_{j=1}^{\delta}\left(1-\frac{1}{i+\frac{j}{\delta}}\right) \prod_{j=1}^{d-\delta}\left(1-\frac{1}{i+\frac{j}{d-\delta}}\right)-\prod_{j=1}^{d}\left(1-\frac{1}{i+\frac{j}{d}}\right) .
$$

Then we only need to show that $Q(i) \geq 0$ for $i \geq 1$. We do this by showing

$$
\left(\frac{1}{d}, \ldots, \frac{d-1}{d}, \frac{d}{d}\right) \preceq\left(\frac{1}{\delta}, \ldots, \frac{\delta-1}{\delta}, \frac{\delta}{\delta}, \frac{1}{d-\delta}, \ldots, \frac{d-\delta-1}{d-\delta}, \frac{d-\delta}{d-\delta}\right),
$$

or equivalently,

$$
\left(\frac{1}{d}, \ldots, \frac{d-1}{d}\right) \preceq\left(\frac{1}{\delta}, \ldots, \frac{\delta-1}{\delta}, \frac{1}{d-\delta}, \ldots, \frac{d-\delta-1}{d-\delta}, 1\right) .
$$

Note that $(d, \delta)=1$ implies all fractions $\left\{\frac{j}{d}\right\}_{j=1}^{d-1},\left\{\frac{j}{\delta}\right\}_{j=1}^{\delta-1}$ and $\left\{\frac{j}{d-\delta}\right\}_{j=1}^{d-\delta-1}$ are different. Hence it suffices to show that every term of $\left\{\frac{j}{\delta}\right\}_{j=1}^{\delta-1} \cup\left\{\frac{j}{d-\delta}\right\}_{j=1}^{d-\delta-1}$ is precisely in one of $d-2$ open intervals $\left(\frac{k}{d}, \frac{k+1}{d}\right)$, where $k=1, \ldots, d-2$. Indeed, neither two terms of $\left\{\frac{j}{\delta}\right\}_{j=1}^{\delta-1}$ nor two terms of $\left\{\frac{j}{d-\delta}\right\}_{j=1}^{d-\delta-1}$ are in the same interval since their difference is larger than $\frac{1}{d}$. On the other hand, if $\frac{j}{\delta}$ and $\frac{j^{\prime}}{d-\delta}$ are in a certain interval $\left(\frac{k}{d}, \frac{k+1}{d}\right)$, then so is $\frac{j+j^{\prime}}{d}$ by Fact 2, which is impossible. Thus there exists precisely one term of $\left\{\frac{j}{\delta}\right\}_{j=1}^{\delta-1} \cup\left\{\frac{j}{d-\delta}\right\}_{j=1}^{d-\delta-1}$ in every open interval $\left(\frac{k}{d}, \frac{k+1}{d}\right)$, as desired. This completes our proof.

For the second generalization of the log-convexity of the central binomial coefficients, we consider sequences of binomial coefficients located along a vertical ray with origin $\left(\begin{array}{c}n_{0} \\ 0\end{array}\right)$ in the Pascal triangle.

Proposition 2. Let $n_{0} \geq 0$ and $V_{i}\left(n_{0}\right)=\left(\begin{array}{c}n_{0}+2 i \\ i\end{array}\right)$. Then $V_{0}\left(n_{0}\right), V_{1}\left(n_{0}\right), \ldots, V_{m}\left(n_{0}\right)$ is log-concave and $V_{m-1}\left(n_{0}\right), V_{m}\left(n_{0}\right), V_{m+1}\left(n_{0}\right), \ldots$ is log-convex, where $m=n_{0}^{2}-\left\lceil\frac{n_{0}}{2}\right\rceil$. 
Proof. The sequence $V_{i}(0)=\left(\begin{array}{c}2 i \\ i\end{array}\right)$ is just the central binomial coefficients and therefore log-convex for $i \geq 0$. It implies that the sequence $V_{i}(1)=\left(\begin{array}{c}1+2 i \\ i\end{array}\right)$ is log-convex for $i \geq 0$ since $V_{i}(1)=\frac{1}{2} V_{i+1}(0)$. Now let $n_{0} \geq 2$ and define $f(i)=V_{i+1}\left(n_{0}\right) / V_{i}\left(n_{0}\right)$ for $i \geq 0$. Then, to show the statement, it suffices to show that

$$
f(0)>f(1)>\cdots>f(m-1) \text { and } f(m-1)<f(m)<f(m+1)<\cdots
$$

for $m=n_{0}^{2}-\left\lceil\frac{n_{0}}{2}\right\rceil$.

By the definition we have

$$
f(i)=\frac{\left(\begin{array}{c}
n_{0}+2(i+1) \\
i+1
\end{array}\right)}{\left(\begin{array}{c}
n_{0}+2 i \\
i
\end{array}\right)}=\frac{\left(n_{0}+2 i+1\right)\left(n_{0}+2 i+2\right)}{(i+1)\left(n_{0}+i+1\right)} .
$$

The derivative of $f(i)$ with respect to $i$ is

$$
f^{\prime}(i)=\frac{2 i^{2}-2\left(n_{0}-2\right)\left(n_{0}+1\right) i-\left(n_{0}+1\right)\left(n_{0}^{2}-2\right)}{(i+1)^{2}\left(n_{0}+i+1\right)^{2}} .
$$

The numerator of $f^{\prime}(i)$ has the unique positive zero

$$
\begin{aligned}
r & =\frac{2\left(n_{0}-2\right)\left(n_{0}+1\right)+\sqrt{4\left(n_{0}-2\right)^{2}\left(n_{0}+1\right)^{2}+8\left(n_{0}+1\right)\left(n_{0}^{2}-2\right)}}{4} \\
& =\frac{\left(n_{0}-2\right)\left(n_{0}+1\right)}{2}+\frac{n_{0} \sqrt{n_{0}^{2}-1}}{2} .
\end{aligned}
$$

It implies that $f^{\prime}(i)<0$ for $0 \leq i<r$ and $f^{\prime}(i)>0$ for $i>r$. Thus we have

$$
f(0)>f(1)>\cdots>f(\lfloor r\rfloor) \text { and } f(\lceil r\rceil)<f(\lceil r\rceil+1)<f(\lceil r\rceil+2)<\cdots .
$$

It remains to compare the values of $f(\lfloor r\rfloor)$ and $f(\lceil r\rceil)$. Note that

$$
\frac{n_{0}^{2}-n_{0} \sqrt{n_{0}^{2}-1}}{2}=\frac{n_{0}}{2\left(n_{0}+\sqrt{n_{0}^{2}-1}\right)}<\frac{1}{2} \text {. }
$$

Hence

$$
\left\lceil\frac{n_{0} \sqrt{n_{0}^{2}-1}}{2}\right\rceil= \begin{cases}\frac{n_{0}^{2}}{2}, & \text { if } n_{0} \text { is even; } \\ \frac{n_{0}^{2}+1}{2}, & \text { if } n_{0} \text { is odd }\end{cases}
$$

and so

$$
\lceil r\rceil=\frac{\left(n_{0}-2\right)\left(n_{0}+1\right)}{2}+\left\lceil\frac{n_{0} \sqrt{n_{0}^{2}-1}}{2}\right\rceil= \begin{cases}n_{0}^{2}-\frac{n_{0}}{2}-1, & \text { if } n_{0} \text { is even; } \\ n_{0}^{2}-\frac{n_{0}+1}{2}, & \text { if } n_{0} \text { is odd }\end{cases}
$$

If $n_{0}$ is even, then by (2) we have

$$
f(\lceil r\rceil)=\frac{16 n_{0}^{2}-8}{4 n_{0}^{2}-1}=4-\frac{4}{4 n_{0}^{2}-1}
$$


and

$$
f(\lfloor r\rfloor)=f(\lceil r\rceil-1)=\frac{16 n_{0}^{4}-40 n_{0}^{2}+16}{4 n_{0}^{4}-9 n_{0}^{2}+4}=4-\frac{4\left(n_{0}^{2}-2\right)}{4 n_{0}^{4}-9 n_{0}^{2}+4} .
$$

Thus $f(\lfloor r\rfloor)>f(\lceil r\rceil)$ since $f(\lfloor r\rfloor)-f(\lceil r\rceil)=\frac{8}{\left(4 n_{0}^{2}-1\right)\left(4 n_{0}^{4}-9 n_{0}^{2}+4\right)}>0$. Also, $\lceil r\rceil=m-1$. Combining (3) we obtain (1).

If $n_{0}$ is odd, then

$$
f(\lceil r\rceil)=4-\frac{4\left(n_{0}^{2}+1\right)}{4 n_{0}^{4}+3 n_{0}^{2}+1}
$$

and

$$
f(\lfloor r\rfloor)=4-\frac{4\left(n_{0}^{2}-1\right)}{4 n_{0}^{4}-5 n_{0}^{2}+1}
$$

It is easy to verify that $f(\lfloor r\rfloor)<f(\lceil r\rceil)$. Also, $\lfloor r\rfloor=\lceil r\rceil-1=m-1$. Thus (1) follows. This completes our proof.

\section{Concluding remarks and open problems}

In this paper we show that the sequence $C_{i}=\left(\begin{array}{l}n_{0}+i d \\ k_{0}+i \delta\end{array}\right)$ is unimodal when $d<\delta$. A further problem is to find out the value of $i$ for which $C_{i}$ is a maximum. Tanny and Zuker [14, 15, 16] considered such a problem for the sequence $\left(\begin{array}{c}n_{0}-i d \\ i\end{array}\right)$. For example, it is shown that the sequence $\left(\begin{array}{c}n_{0}-i \\ i\end{array}\right)$ attains the maximum when $i=\left\lfloor\left(5 n_{0}+7-\sqrt{5 n_{0}^{2}+10 n_{0}+9}\right) / 10\right\rfloor$. Let $r\left(n_{0}, d\right)$ be the least integer at which $\left(\begin{array}{c}n_{0}-i d \\ i\end{array}\right)$ attains its maximum. They investigated the asymptotic behavior of $r\left(n_{0}, d\right)$ for $d \rightarrow \infty$ and concluded with a variety of unsolved problems concerning the numbers $r\left(n_{0}, d\right)$. An interesting problem is to consider analogue for the general binomial sequence $C_{i}=\left(\begin{array}{l}n_{0}+i d \\ k_{0}+i \delta\end{array}\right)$ when $d<\delta$. It often occurs that unimodality of a sequence is known, yet to determine the exact number and location of modes is a much more difficult task.

A finite sequence of positive numbers $a_{0}, a_{1}, \ldots, a_{n}$ is called a Pólya frequency sequence if its generating function $P(x)=\sum_{i=0}^{n} a_{i} x^{i}$ has only real zeros. By the Newton's inequality, if $a_{0}, a_{1}, \ldots, a_{n}$ is a Pólya frequency sequence, then

$$
a_{i}^{2} \geq a_{i-1} a_{i+1}\left(1+\frac{1}{i}\right)\left(1+\frac{1}{n-i}\right)
$$

for $1 \leq i \leq n-1$, and the sequence is therefore log-concave and unimodal with at most two modes (see Hardy, Littlewood and Pólya [9, p. 104]). Darroch [6] further showed that each mode $m$ of the sequence $a_{0}, a_{1}, \ldots, a_{n}$ satisfies

$$
\left\lfloor\frac{P^{\prime}(1)}{P(1)}\right\rfloor \leq m \leq\left\lceil\frac{P^{\prime}(1)}{P(1)}\right\rceil .
$$

We refer the reader to $[3,4,8,11,12,13,20]$ for more information.

For example, the binomial coefficients $\left(\begin{array}{l}n \\ 0\end{array}\right),\left(\begin{array}{l}n \\ 1\end{array}\right), \ldots,\left(\begin{array}{l}n \\ n\end{array}\right)$ is a Pólya frequency sequence with the unique mode $n / 2$ for even $n$ and two modes $(n \pm 1) / 2$ for odd $n$. On the other 
hand, the sequence $\left(\begin{array}{c}n \\ 0\end{array}\right),\left(\begin{array}{c}n-1 \\ 1\end{array}\right),\left(\begin{array}{c}n-2 \\ 2\end{array}\right), \ldots,\left(\begin{array}{l}{[n / 2\rceil} \\ {[n / 2\rfloor}\end{array}\right)$ is a Pólya frequency sequence since its generating function is precisely the matching polynomial of a path on $n$ vertices. Hence we make the more general conjecture that every sequence of binomial coefficients located in a transversal of the Pascal triangle is a Pólya frequency sequence.

Conjecture 2. Let $C_{i}=\left(\begin{array}{l}n_{0}+i d \\ k_{0}+i \delta\end{array}\right)$ where $n_{0} \geq k_{0}$ and $\delta>d>0$. Then the finite sequence $\left\{C_{i}\right\}_{i}$ is a Pólya frequency sequence.

In Proposition 2 we have shown that the sequence $V_{i}\left(n_{0}\right)=\left(\begin{array}{c}n_{0}+2 i \\ i\end{array}\right)$ is first log-concave and then log-convex. It is possible that an arbitrary sequence of binomial coefficients located along a ray in the Pascal triangle has the same property as the sequence $V_{i}\left(n_{0}\right)$. We leave this as a conjecture to end this paper.

Conjecture 3. Let $C_{i}=\left(\begin{array}{l}n_{0}+i d \\ k_{0}+i \delta\end{array}\right)$ where $n_{0} \geq k_{0}$ and $d>\delta>0$. Then there is a nonnegative integer $m$ such that $C_{0}, C_{1}, \ldots, C_{m-1}, C_{m}$ is log-concave and $C_{m-1}, C_{m}, C_{m+1}, \ldots$ is log-convex.

\section{Acknowledgments}

The authors thank Feng Guo, Po-Yi Huang and Yeong-Nan Yeh for helpful discussions.

\section{References}

[1] H. Belbachir, F. Bencherif and L. Szalay, Unimodality of certain sequences connected with binomial coefficients, J. Integer Seq. 10 (2007), Article 07. 2. 3.

[2] M. Bóna and B. Sagan, Two injective proofs of a conjecture of Simion, J. Combin. Theory Ser. A 10 (2003) 79-89.

[3] F. Brenti, Unimodal, log-concave, and Pólya frequency sequences in combinatorics, Mem. Amer. Math. Soc. 413 (1989).

[4] F. Brenti, Log-concave and unimodal sequences in algebra, combinatorics, and geometry: An update, Contemp. Math. 178 (1994) 71-89.

[5] L. Comtet, Advanced Combinatorics, Reidel, Dordrecht, 1974.

[6] J. N. Darroch, On the distribution of the number of successes in independent trials, Ann. Math. Statist. 35 (1964) 1317-1321.

[7] T. Došlić and D. Veljan, Logarithmic behavior of some combinatorial sequences, Discrete Math. 308 (2008) 2182-2212.

[8] P. Erdős, On a conjecture of Hammersley, J. London Math. Soc. 28 (1953) 232-236.

[9] G. H. Hardy, J. E. Littlewood and G. Pólya, Inequalities, Cambridge University Press, Cambridge, 1952.

[10] L. L. Liu and Y. Wang, On the log-convexity of combinatorial sequences, Adv. in. Appl. Math. 39 (2007) 453-476. 
[11] L. L. Liu and Y. Wang, A unified approach to polynomial sequences with only real zeros, Adv. in Appl. Math. 38 (2007) 542-560.

[12] S.-M. Ma and Y. Wang, $q$-Eulerian polynomials and polynomials with only real zeros, Electron. J. Combin. 15 (2008), Research Paper 17, 9 pp.

[13] R. P. Stanley, Log-concave and unimodal sequences in algebra, combinatorics and geometry, Ann. New York Acad. Sci. 576 (1989) 500-534.

[14] S. Tanny and M. Zuker, On a unimodal sequence of binomial coefficients, Discrete Math. 9 (1974) 79-89.

[15] S. Tanny and M. Zuker, On a unimodal sequence of binomial coefficients II, J. Combin. Inform. System Sci. 1 (1976) 81-91.

[16] S. Tanny and M. Zuker, Analytic methods applied to a sequence of binomial coefficients, Discrete Math. 24 (1978) 299-310.

[17] Y. Wang, A simple proof of a conjecture of Simion, J. Combin. Theory Ser. A 100 (2002) 399-402.

[18] Y. Wang, Proof of a conjecture of Ehrenborg and Steingrmsson on excedance statistic, European J. Combin. 23 (2002) 355-365.

[19] Y. Wang, Linear transformations preserving log-concavity, Linear Algebra Appl. 359 (2003) 161-167.

[20] Y. Wang and Y.-N. Yeh, Polynomials with real zeros and Pólya frequency sequences, J. Combin. Theory Ser. A 109 (2005) 63-74.

[21] Y. Wang and Y.-N. Yeh, Proof of a conjecture on unimodality, European J. Combin. 26 (2005) 617-627.

[22] Y. Wang and Y.-N. Yeh, Log-concavity and LC-positivity, J. Combin. Theory Ser. A 114 (2007) 195-210. 